\title{
Estimation Method
}

National Cancer Institute

\section{Source}

National Cancer Institute. Estimation Method. NCI Thesaurus. Code C81307.

A description of the technique for the approximate calculation of a value. 